\title{
A rare cause of acute abdomen: torsion of a second spleen
}

\author{
S Gobishangar, S Raviraj \\ Ceylon Medical Journal 2013; 58: 34-35
}

\section{Introduction}

Congenital abnormalities of the spleen are not uncommon. Commonest abnormality is the occurrence of accessory spleens (splenunculi). However the presence of a second spleen is very rare. While the size and shape of an accessory spleen can vary markedly, a second spleen is almost identical to the normal spleen morphologically. Torsion in a second spleen is a rare clinical entity and its diagnosis is very difficult. It is due to abnormal anatomical position with a long pedicle and absence or laxity of suspensory ligaments compared to the normal spleen [1].

\section{Case report}

A 12-year old previously healthy girl was admitted to the hospital with two days of upper abdominal pain. The pain was constant and moderate and was not associated with nausea or vomiting. Her bowel habits were normal prior to this illness.

On examination her temperature was $37.5^{\circ} \mathrm{C}$, blood pressure was $90 / 60 \mathrm{mmHg}$ and the pulse rate was $92 / \mathrm{min}$. The abdomen was slightly distended with tenderness, rigidity and guarding. The abdominal signs were more marked in the epigastrium and left upper quadrant.

Laboratory investigations were normal except for leukocytosis. The X-ray abdomen and erect chest X-ray were normal. Abdominal ultrasound scan revealed a solid mass in the epigastric region. Liver, spleen, gall bladder and kidneys were normal.

At surgery a haemorrhagic, infarcted second spleen was located in the greater omentum, identical to the normal spleen morphologically. This second spleen was about $7.0 \times 4.0 \times 3.5 \mathrm{~cm}$ with a long pedicle. The normal spleen was situated in the expected anatomical position (Figures 1 and 2). The removed second spleen showed infarcted, necrotic spleen tissues and thrombosis of the hilar vessels microscopically. Polyvalent pneumococcal vaccine was not administered postoperatively due to the presence of a normal spleen.

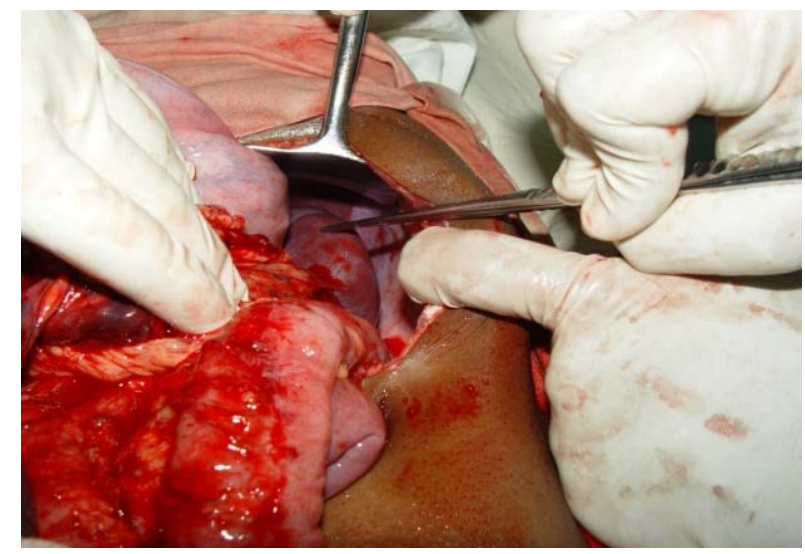

Figure 1. The normal spleen in the left hypochondrium.

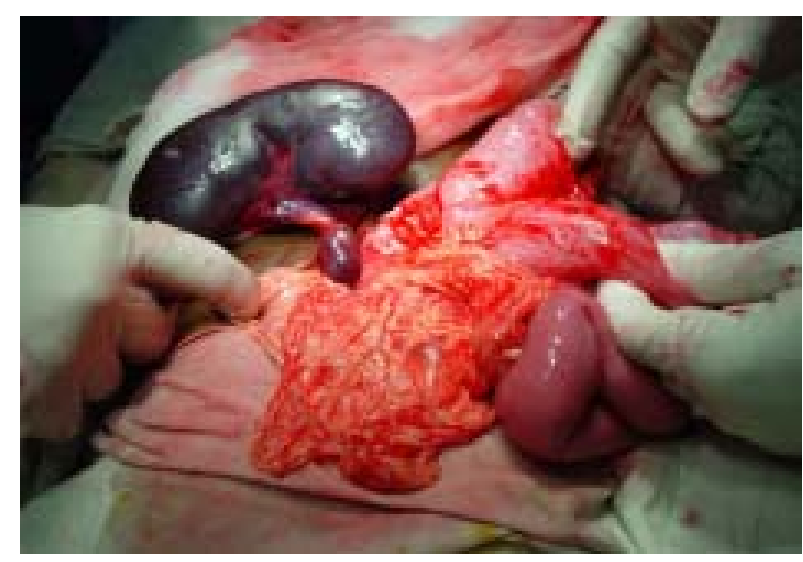

Figure 2. Accessory spleen in the greater omentum with a long twisted pedicle and with no supporting ligaments.

\section{Discussion}

The spleen develops in the left hypochondrium of the abdomen from the mesenchymal cells of dorsal mesogastrium. The gastrosplenic, splenocolic, splenophrenic and splenorenal ligaments maintain the spleen in its anatomical position. The congenital absence or laxity 
of these ligaments facilitate the free mobility of the spleen and allow the splenic pedicle to elongate predisposing to torsion.

Accessory spleen (supernumerary spleen, splenule or splenunculus) is usually a small nodule of splenic tissue found apart from the main body of the spleen. An accessory spleen is found in $10 \%$ of population [2]. They form either by the result of developmental anomalies or trauma [3]. But there is little information available about the second spleen. The typical size of an accessory spleen may vary from 1 to $3 \mathrm{~cm}$ and can take any shape [1]. This girl had a second spleen which is identical in size and morphology to the normal spleen.

Acute complete splenic torsion may present as fever, signs of peritonitis and pressure symptoms on other organs $[3,4]$. The consequences of splenic torsion can be dangerous. The occlusion of splenic vessels may lead to haemorrhagic infarction of the entire spleen, splenic gangrene, perisplenic adhesions and splenic cysts or abscess [4]. Abdominal ultrasound, CT, scintigraphy and angiography have been used for the diagnosis of splenic torsion. Preoperative diagnosis is usually difficult and the diagnosis is made only at laparotomy.

\section{References}

1. Gayer G, Zissin R, Apter S, et al. CT findings in congenital anomalies of the spleen. British Journal of Radiology 2001; 74: 767-72.

2. Keith L Moore. Clinically oriented anatomy. 3rd edition. Baltimore: Williams \& Wilkins, 1992.

3. Michaels L. Spontaneous torsion of spleen involving tail of Pancreas. Lancet 1954; 267: 23.

4. Frederik HB, Khettry J, Filler RM. Splenic torsion and accessory spleen. Journal of Paediatric Surgery 1975; 10: 913.

\section{Isolation of Neisseria meningitidis from cadaveric blood}

\section{M B T Dissanayake, P C L S Waduge, N S Chandrasiri, R C Ediriweera}

Ceylon Medical Journal 2013; 58: 35-36

\section{Introduction}

We describe a case of sudden death of a $2 \frac{1}{2}$ year old boy with one day fever and rash due to meningococcal septicaemia. The child was admitted to the Colombo South Teaching Hospital in a state of shock. There was a purpuric rash all over the body. Immediate resuscitation was started but the child developed a cardiac arrest within minutes of admission. Resuscitation failed and death was confirmed at $11.55 \mathrm{pm}$ with a probable diagnosis of meningococcaemia.

According to the mother, child developed mild fever and vomiting in the morning. By evening he developed high fever and a few patches in the face which made them take the child to a doctor. He was given antipyretics and sent home. The skin patches evolved to involve the whole body and the child was brought to hospital around 10.15 pm with difficulty in breathing. Parents were concerned about a possible anaphylactic drug reaction to a drug that was given to him by the doctors. A post-mortem examination carried out on the following day revealed haemorrhages in adrenals, brain and lungs (Figures 1 and 2). There were no features of meningitis or laryngeal oedema. Cardiac blood and cerebro-spinal fluid (CSF) were collected aseptically. CSF was blood stained and Gram stain failed to reveal organisms.

After 24 hours of incubation, cultures yielded a growth and the isolate was confirmed as Neisseria meningitidis by standard biochemical tests [1]. The household members and the hospital staff who were at risk were given appropriate chemoprophylaxis against meningococcal infection [2].

It is interesting to note that the isolate from a cadaver which was in the freezer for 14 hours yielded an organism which is supposed to be susceptible to low temperatures. Hence it is worth attempting to culture the meningococcus from cadaveric blood which would be crucial for medicolegal issues and preventive measures among contacts.

Colombo South Teaching Hospital, Kalubowila, Sri Lanka.

Correspondence: DMBTD, e-mail: <thushari.dissanayake@y7mail.com>. Received 17 May and revised version accepted 25 October 2012. Competing interests: none declared. 